\title{
Factores que Afectan la Duración de los Tratamientos de Ortodoncia en un Servicio Público de Salud
}

\author{
Factors that Affect the Duration of Orthodontic Treatment in Public Health Services
}

Carlos Felipe Zepeda Diaz

ZEPEDA, D. C. F. Factores que afectan la duración de los tratamientos de ortodoncia en un Servicio Público de Salud. Int. J. Odontostomat., 13(3):321-324, 2019.

RESUMEN: La duración de los tratamientos de ortodoncia siempre ha sido una de las mayores preocupaciones, tanto en los pacientes como en los Ortodoncistas. El proposito de esta investigacion fue determinar la magnitud de las inasistencias a los controles por parte de los pacientes y como estas y otras variables, influyen en la duración de los tratamientos de Ortodoncia en el Centro de Salud Familiar (CESFAM) Dr. Victor Manuel Fernandez, Servicio de Salud de Concepcion, Chile. Se realizo un estudio descriptivo, de tipo transversal, retrospectivo, en el que se utilizaron las fichas clínicas de todos los pacientes que fueron dados de alta por los Ortodoncistas del CESFAM Dr. V. M. F. durante el año 2017. La tabulación se realizó en Microsoft Excel y el análisis estadístico se hizo en InfoStat 17. Se incluyeron 246 pacientes, con una edad de ingreso promedio de 13,6 años. La maloclusion predominante fue la Clase I de Angle (71\%). Estos pacientes tuvieron una duracion de tratamiento promedio de 33,9 meses. Se concluyó que a mayor cantidad de inasistencias, el tratamiento de ortodoncia tuvo una duración mayor.

PALABRAS CLAVE: ortodoncia, duración de tratamiento, efectividad del tratamiento.

\section{INTRODUCCIÓN}

Durante el desarrollo de la ortodoncia a través de los años, se ha avanzado considerablemente en mejorar los aparatos, insumos y técnicas empleadas, con el fin de tener tratamientos más eficaces y cortos en el tiempo de duración. La literatura científica disponible describe como promedio de duración una media que oscila entre los 20 y 30 meses para finalizar tratamientos de Ortodoncia.

En la Salud Publica moderna, la eficiencia en los costos en un importante objetivo, por lo que un tratamiento que se extiende demasiado en el tiempo atenta contra el objetivo antes mencionado, por lo que los esfuerzos clínicos deben estar orientados a ser eficaces. A su vez, tratamientos más cortos disminuyen la probabilidad de efectos secundarios indeseables como pueden ser reabsorciones radiculares, y problemas inducidos por acumulacion de placa bacteriana como manchas blancas, caries o enfermedades periodontales.

El propósito de este estudio fue determinar la duración que tienen los tratamientos de Ortodoncia en el CESFAM Dr. Víctor Manuel Fernández del Servicio de Salud de Concepción, y la magnitud de las inasistencias a los controles por parte de los pacientes, y como estas y otras variables, influyen en el alargamiento de los mismos.

\section{MATERIAL Y METODO}

Estudio descriptivo, de tipo transversal, retrospectivo, de pacientes atendidos por el Servicio de Ortodoncia del CESFAM Dr. Victor Manuel Fernandez, Chile. Este estudio recibio la aprobacion del comité de etica del Servicio de Salud de Concepcion. Se trabajo con las fichas clínicas de todos los pacientes que fueron dados de alta por los Ortodoncistas del CESFAM Dr. V.M.F. durante el año 2017. Para el analisis se consideraron las siguientes variables: Edad, Sexo, tipo de maloclusion (segun Angle), indicacion de extraccion dentaria, cantidad de inasistencias a control y duracion total del tratamiento. 
Los datos fueron codificados y tabulados en Microsoft Excel 2016 (Microsoft Corp, USA) de acuerdo al protocolo respectivo, sin incluir la identificación personal. El análisis incluyó una exploración de los datos con generación de estadística descriptiva con medidas de tendencia central y de dispersión (medias con su desviación estándar) para las variables cuantitativas, y tablas de frecuencias y gráficos para las variables cualitativas. Para comparar medias se utilizó prueba $t$ y ANOVA con comparaciones múltiple. Los contrastes se consideraron significativos al $5 \%$. El software utilizado para el análisis fue InfoStat 17.

\section{RESULTADOS}

La población total de análisis de este estudio consistió en 246 pacientes. La edad promedio de comienzo de tratamiento fue de 13,8 años, mientras que la edad promedio de alta de tratamiento fue de 16,7 años. El numero de fallas a las citaciones fue en promedio 3,1 , con un rango de 0-15.

Las mujeres representaron el $54 \%$ (134) de la población estudiada, mientras que los hombres fueron el $46 \%$ (112).

El mayor porcentaje de usuarios atendidos en el Consultorio correspondió a pacientes que presentan Maloclusión Clase I de Angle (71\%), seguidos de las Clase II $(27 \%)$ y la Clase III (2\%).

Los pacientes presentaron la siguiente distribución, si tuvieron al menos 1 ausencia (o más) durante su tratamiento ( $83 \%$ ), mientras que los pacientes que tuvieron asistencia completa a sus citaciones llegaron solo al $17 \%$.

En los tratamientos de Ortodoncia se indicó un $63 \%$ de extracciones para la planificación de sus tratamientos. Se estableció una media de 2,25 dientes extraídos por pacientes.
En general, los tratamiento de ortodoncia tuvieron un promedio de duración general de 33,9 meses, teniendo en los hombres un promedio de duración de 34,1 meses y en las mujeres 33,6 , no existiendo una diferencia estadísticamente significativa según el sexo.

En la Figura 6, se muestra la duración de los tratamientos de Ortodoncia se vio afectada según si se indicó o no extracciones para la solución del problema Ortodóncico. En pacientes que no tuvieron exodoncias el tiempo promedio de duración fue de 29 meses mientras que en los pacientes que tuvieron indicación de extracción el promedio fue de 37, por lo que en estos casos los tratamiento duran en promedio 8 meses más.

Se extrajeron un total de 554 dientes, existiendo pacientes en los cuales no se extrajeron dientes, mientras que en otros casos se realizaron hasta 8 extracciones en un mismo paciente.

Mientras mayor es el numero de ausencias a las citaciones de control de ortodoncia, la duración del tratamiento aumenta de manera significativa, lo que se hace ampliamente superior cuando los pacientes acumulan mas de 4 ausencias (el tiempo de tratamiento aumenta en 18 meses mas). (Fig. 1)

Al realizar pruebas múltiples, el rango 3 (4 o más ausencias durante el tratamiento) presentó una diferencia estadísticamente significativa respecto a los otros rangos estudiados (Tabla I). El tratamiento más extenso duró 101 meses, mientras que el más corto solo 8 meses.

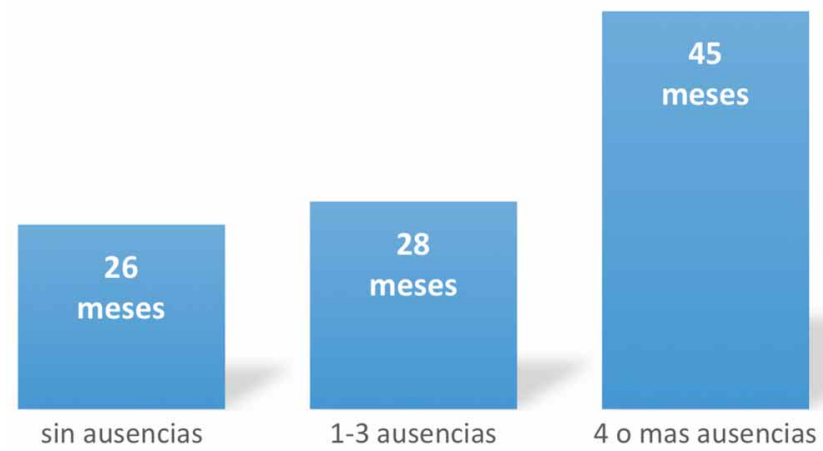

Fig. 1. Duración de tratamiento según rango de ausencias.

Tabla I. Comparación de duración de tratamiento por rango de ausencia.

\begin{tabular}{lllllll}
\hline Rango Ausencias & $\mathrm{N}$ & Media & $\mathrm{P}^{*}$ & $\mathrm{D} . \mathrm{E}$ & Min. & Max. \\
\hline 0 & 44 & 26,0 & & 12,5 & 10,0 & 66,0 \\
$1-3$ & 109 & 28,0 & $<0,0001$ & 11,2 & 8,0 & 78,0 \\
4 o mas & 93 & 44.5 & & 20,3 & 15,0 & 101,0 \\
\hline
\end{tabular}

*p: prueba ANOVA; n: población; D.E.: Desviación estándar. 


\section{DISCUSIÓN}

Este estudio analizo datos de ortodoncia con la poblacion objetivo (adolescentes) del Servicio Público de Salud de Chile, aunque la literaura disponible permite concluir que la edad no juega un rol critico en la duracion de los tratamientos como lo concluido por Mabreas et al. (2008), Bichara et al. (2016), Dyer et al. (1991) y Robb et al. (1998).

El promedio de duracion de los tratamientos de Ortodoncia en el Servicio Dental del CESFAM Dr. Víctor Manuel Fernández encontrada en esta investigación fue de un 33,9 meses, lo que es un resultado mayor a lo encontrado por la mayoria de los autores como Grewe \& Hermanson (1973) con 23,1 meses, o en las revisiones sitematicas de Tsichlaki et al. (2016) que encontró una duración de 20,22 meses o Papageorgiou et al. (2017) con 24,9 meses. El unico estudio analizado con una duracion de tratamiento similar fue el de Melo et al. (2013) con 32,2 meses, aunque su poblacion de analisis eran solo adultos. La explicacion a las diferencias encontradas con la mayoria de los estudios, se atribuyen a 2 factores principalmente, el gran numero de ausencias a los controles y la alta frecuencia de indicacion de extraccion a los tratamientos de ortodoncia.

Como esta documentado en la literatura y en este estudio, no se encontraron diferencias en la duracion de los tratamientos según si el paciente fue de sexo masculino o femenino. Una excepcion es lo expuesto por Skidmore et al. (2006), aunque la diferencia ( 1,2 meses mayor en hombres) no es considerada significativa.

La distribución de los pacientes por tipo de maloclusion encontrada (según los criterios de Angle) es concordante con lo reportado por otros autores como Beckwith et al. (1999), Burgos (2014) y Al Jadidi et al. (2018), aunque la maloclusion de Clase III encontrada es menor a otros estudios, lo que se explica probablemente porque los casos complejos de resolucion quirurgica no se atienden en el CESFAM.

La gran mayoría de los pacientes (83\%) se ausento al menos 1 vez durante el desarrollo de su tratamiento, lo que es concordante con lo reportado por Trenouth (2003), e implicó discontinuidad en el tratamiento de Ortodoncia, perdida de horas profesional, caída de Brackets, etc., todos estos factores en mayor o menor medida provocaron alargamientos exce- sivos en la duración del tratamiento para finalizar los casos analizados, situación que atenta contra los principios de eficiencia que debe presentar un servicio de alta demanda como el de la especialidad de Ortodoncia (amplia lista de espera de pacientes). Finalmente, el compromiso del paciente (medido como la cantidad de ausencias que presenta durante su tratamiento de ortodoncia) se definio como el principal factor que afecto la duracion de los tratamientos en este estudio, lo que es concordante con lo concluido por varios autores como Bichara et al., Melo et al. y Robb et al. Esta conclusión sugiere que pacientes poco comprometidos no deben iniciar un tratamiento de Ortodoncia, o en caso de iniciarlo deben tener un seguimiento mas estricto para no perder tiempo y recursos en el desarrollo de su tratamiento.

Se indicó extracción dentaria en un $63 \%$ de los casos, ademas se extrajeron una media de 2,25 dientes por paciente, existiendo una duración promedio mas larga en los tratamientos que tuvieron indicación de exodoncia (8 meses mas de duración) versus los que no presentaron extracciones en su planificación de tratamiento, lo que se explica porque los casos con extracciones generalmente tienen una complejidad mayor e involucran mayor movimiento dentario. La investigacion de Alger (1988) y de Buschang et al. (2012) concluyeron en su analisis un resultado similar a este trabajo, aunque la diferencia en la duracion era solo de 4 meses, lo mismo que Fink \& Smith (1992) con 5 meses de diferencia. La excepcion a esta tendencia encontrada en la bibliografia fue fue un estudio realizado por Vig et al. (1990) que no encontro diferencias en el tiempo de duracion según la indicacion de extraccion.

Los tratamientos de Ortodoncia tienen una larga duración, lo que esta definido por una multiplicidad de factores, por lo que es imperativo un asertado diagnostico y una buena planificación del tratamiento por parte del profesional, con un uso adecuado de las herramientas y tecnologias disponibles, y un gran compromiso por parte del paciente (definido en la asistencia a sus controles, cuidado de los aparatos y mantencion de una buena higiene oral) para lograr una resolución satisfactoria al final del tratamiento, con un eficiente uso de los recursos disponibles, situacion que es especialmente critica en un Servicio Publico de Salud.

AGRADECIMIENTOS. A la Dra. Carmen Gloria Thibaut, Jeja del Servicio Dental del CESFAM Dr. Victor Manuel Fernandez, por su motivacion en mejorar nues- 
tra labor diaria y su apoyo en la realizacion de este trabajo. A Camila Oviedo y Rocio Vargas por la ayuda en la recoleccion inicial de datos y al profesor Luis Luengo por su invaluable ayuda en el analisis estadistico de este trabajo.

ZEPEDA, D. C. F. Factors that affect the duration of orthodontics treatment in a public health services. Int. J. Odontostomat., 13(3):321-324,2019.

ABSTRACT: The duration of orthodontic treatments has always been one of the biggest concerns, both in patients and for orthodontists. The purpose of this investigation was to determine the magnitude of the absences to the controls of the patients and how they influence the duration of the orthodontic treatments in the CESFAM Dr. Victor Manuel Fernandez, Concepcion, Chile. Adescriptive, cross-sectional, retrospective study was carried out, in which the clinical records of all patients discharged by the Orthodontists of CESFAM Dr. V.M.F. during the year 2017 were analyzed. The tabulation was performed in Microsoft Excel and the statistical analysis was carried out in InfoStat 17. In total, 246 patients were included, with an average age of admission of 13.6 years. The predominant malocclusion was Class I of Angle $(71 \%)$. These patients had an average treatment duration of 33.9 months. It was further concluded that the length of orthodontic treatment, was also affected by the increased number of times patients failed to show for treatment.

KEY WORDS: orthodontics, treatment duration, treatment effectiveness.

\section{REFERENCIAS BIBLIOGRÁFICAS}

Al Jadidi, L.; Sabrish, S.; Shivamurthy, P. G. \& Senguttuvan, V. The prevalence of malocclusion and orthodontic treatment need in Omani adolescent population. J. Orthod. Sci., 7:21, 2018.

Alger, D. W. Appointment frequency versus treatment time. Am. J. Orthod. Dentofacial Orthop., 94(5):436-9, 1988.

Beckwith, F. R.; Ackerman, R. J. Jr.; Cobb, C. M. \& Tira, D. E. An evaluation of factors affecting duration of orthodontic treatment. Am. J. Orthod. Dentofacial Orthop., 115(4):439-47, 1999.

Bichara, L. M.; Aragón, M. L.; Brandão, G. A. \& Normando, D. Factors influencing orthodontic treatment time for non-surgical Class III malocclusion. J. Appl. Oral Sci., 24(5):431-6, 2016.

Burgos, D. Prevalence of malocclusion in 6 to 15 -year-old children and adolescents in Frutillar, Chile. Int. J. Odontostomat., 8(1):139, 2014.

Buschang, P. H.; Campbell, P. M. \& Ruso, S. Accelerating tooth movement with corticotomies: is it possible and desirable? Semin. Orthod., 18(4):286-94, 2012.

Dyer, G. S.; Harris, E. F. \& Vaden, J. L. Age effects on orthodontic treatment: adolescents contrasted with adults. Am. J. Orthod. Dentofacial Orthop., 100(6):523-30, 1991.

Fink, D. F. \& Smith, R. J. The duration of orthodontic treatment. Am. J. Orthod. Dentofacial Orthop., 102(1):45-51, 1992.
Grewe, J. M. \& Hermanson, P. C. Influence of severity of malocclusion on the duration of orthodontic treatment. Am. J. Orthod. Dentofacial Orthop., 63(5):533-6, 1973.

Mabreas, D. \& Athanasiou, A. E. Factors affecting the duration of orthodontic treatment: a systematic review. Eur. J. Orthod., 30(4):386-95, 2008

Melo, A. C.; Carneiro, L. O.; Pontes, L. F.; Cecim, R. L.; de Mattos, J. N. \& Normando, D. Factors related to orthodontic treatment time in adult patients. Dental Press J. Orthod., 18(5):59-63, 2013.

Papageorgiou, S. N.; Höchli, D. \& Eliades, T. Outcomes of comprehensive fixed appliance orthodontic treatment: A systematic review with meta-analysis and methodological overview. Korean J. Orthod., 47(6):401-13, 2017.

Robb, S. I.; Sadowsky, C.; Schneider, B. J. \& BeGole, E. A. Effectiveness and duration of orthodontic treatment in adults and adolescents. Am. J. Orthod. Dentofacial Orthop., 114(4):383-6, 1998.

Skidmore, K. J.; Brook, K. J.; Thomson, W. M. \& Harding, W. J. Factors influencing treatment time in orthodontic patients. $\mathrm{Am}$. J. Orthod. Dentofacial Orthop., 129(2):230-8, 2006.

Trenouth, M. J. Do failed appointments lead to discontinuation of orthodontic treatment? Angle Orthod., 73(1):51-5, 2003.

Tsichlaki, A.; Chin, S. Y.; Pandis, N. \& Fleming, P. S. How long does treatment with fixed orthodontic appliances last? A systematic review. Am. J. Orthod. Dentofacial Orthop., 149(3):308-18, 2016.

Vig, P. S.; Weintraub, J. A.; Brown, C. \& Kowalski, C. J. The duration of orthodontic treatment with and without extractions: a pilot study of five selected practices. Am. J. Orthod. Dentofacial Orthop., 97(1):45-51, 1990.

Dirección para correspondencia:

Dr. Carlos Felipe Zepeda Díaz

Especialista en Ortodoncia

Universidad de Concepción

Concepción

CHILE

Email: ortodonciazepeda@gmail.com

Recibido : 27-12-2018

Aceptado: 02-04-2019 